\title{
Toxicological Studies on Heavy Metal Bioaccumulation and Oxidative DNA Damage in Residents of a Gas Flaring Community
}

\author{
Ifenkwe John Chimerenka ${ }^{1}$, Nwanjo Harrison Ugochukwu ${ }^{2}$, Nwosu Dennis Chimezie ${ }^{3}$, Ofor Igri Bassey ${ }^{4}$ \\ ${ }^{1}$ Health Check Integrated Services, 193 Chief Melford Okilo Way, Onopa, Yenagoa, Bayelsa State, Nigeria \\ healthcheck.hc[at]gmail.com, +2348036662605 \\ ${ }^{2,3}$ Imo State University, Owerri, Nigeria \\ ${ }^{4}$ Federal Medical Centre, Yenagoa, Nigeria
}

\begin{abstract}
This study was carried out on blood samples collected from 200 residents each from Imiringi (Gas Flaring Community) and Odi (Non-Gas Flaring Community) all in Bayelsa State, Nigeria to determine the blood levels of Cadmium, Chromium, Lead, Mercury and Selenium as well as the oxidative DNA damage marker 8-Hydroxy-2-deoxyguanosine (8-OHdG). Blood samples were also analyzed for WBC, Hb, platelets, prothrombin time and serum concentrations of AST, ALT, albumin, ALP, bilirubin, gamma GT, total protein, sodium, potassium, chloride, bicarbonate, urea and creatinine. Residents in Imiringi recorded the highest blood levels of Mercury, Lead, Selenium, Cadmium, Chromium and 8-OHdG and they were all statistically significantly different at p<0.05. Residents in Imiringi had the highest mean values of all the measured liver and kidney functions parameters with the exception of albumin, total protein, sodium, chloride and bicarbonate and these values were all statistically different at $P<0.05$. There was statistically significant difference in the levels of $\mathrm{Hb}, \mathrm{WBC}$ and Platelet while the length of bleeding time was prolonged in the residents of Imiringi community. The findings of this study suggest that the probability of occurrence of diseases associated with metal toxicity and oxidative stress might be higher among residents in the gas flaring community.
\end{abstract}

Keywords: Heavy Metal, Gas Flaring, Bioaccumulation, Oxidative Stress

\section{Introduction}

Bayelsa State in the Niger Delta region of Southern Nigeria is a centre of oil and gas production as well as allied activities. These activities include drilling, storage, refining and transportation of products. In the course of these activities, a large amount of petroleum products and its wastes are discharged into the surrounding environments. Crude oil contains different proportions of heavy metals namely Zinc $(\mathrm{Zn})$, Lead $(\mathrm{Pb})$ Manganese $(\mathrm{Mn})$, Chromium (Cr), Cadmium (Cd), Iron (Fe), Nickel $(\mathrm{Ni})$, Cobalt $(\mathrm{Co})$, Vanadium $(\mathrm{Vd})$, Mercury $(\mathrm{Hg})$, Copper $(\mathrm{Cu})$,Molybdenum (Mo) and Selenium $(\mathrm{Se})$ with Nigeria crude oils having relatively high concentrations of $\mathrm{Fe}, \mathrm{Zn}$, $\mathrm{Cu}, \mathrm{Pb}$ and $\mathrm{Hg}[1]$.

These heavy metals unlike organic contaminants are not degraded further and also cannot decompose into other chemicals with time [2]. This results to their bioaccumulation in the ecosystem, agriculture and the human body.

\section{Literature Review}

Heavy metals exhibit toxicity at low concentrations and the toxic effects can be long lasting due to their accumulation in the biota.A peculiar feature of these xenobiotics is the induction of oxidative stress [3].

Oxidative Stress is a condition characterized by an imbalance between the concentration of Reactive Oxygen Species (ROS) and antioxidants. Excessive accumulation of ROS will lead to cell injury due to damage to nuclear materials (DNA/RNA), proteins, lipid membranes or by oxidatively inactivating specific enzymes by oxidation of Co-factors [4].

Gas flaring which is the burning of natural gas that is associated with crude oil when it is pumped up from the ground is employed in petroleum-producing areas where insufficient investment was made in infrastructure to utilize natural gas to dispose of this associated gas. Nigeria flares about 17.2 billion $\mathrm{cm}^{3}$ of natural gas every year in conjunction with the exploration of crude oil in the Niger Delta [5]. This high level of gas flaring is equal to approximately one quarter of the current gas consumption of the African continent.

\section{Statement of the Problem}

Even though the Niger Delta area has grown to be fairly dependent on oil and has equally become the center of current industrial development and economic activities, considerations are rarely made on how oil exploration processes create environmental, health, and social problems in local communities near oil producing fields. Gas flaring contributes to climate change, which has serious implications for both Nigeria and the rest of the world. The burning of fossil fuel, mainly coal, oil and gas has led to global warming and is projected to get worse during the course of the 21st century according to the Intergovernmental Panel on Climate Change (IPCC) due to the emission of carbon dioxide, the main greenhouse gas [5].

Gases such as Carbon Monoxide (CO), Nitrogen Oxides (NO), Hydrogen Sulfide $\left(\mathrm{H}_{2} \mathrm{~S}\right)$, volatile organic compounds, photochemical oxidants and other harmful 


\section{International Journal of Science and Research (IJSR) \\ ISSN (Online): 2319-7064}

Index Copernicus Value (2016): 79.57 | Impact Factor (2015): 6.391

compounds are contained in flared gases [6]. Skin and eye irritations as well as respiratory tract infections such as pneumonia are associated with exposure to these gases. Water sources from gas flared areas have raised levels of conductivity, total dissolved solids, total hardness, temperature, dissolved oxygen, chemical oxygen demand, chloride, nitrate, nitrites, sulphates, cadmium, lead, potassium and zinc above the WHO acceptable limits for drinking water [7]. This makes water from these sources unsuitable for human consumption.

From the foregoing, it becomes expedient to assess the levels of heavy metals and oxidative stress markers in residents of these communities who are exposed to the flared gases, drink from the polluted water, eat plants harvested from the surrounding soil and equally consume aquatic species found in these environments. Bearing in mind the cumulative health effects that may result from these, this study tried to explore the impact of gas flaring on human health to improve mitigation efforts and also to prevent adverse health effects on individuals living in affected communities.

\section{Materials and Methods}

\section{Study Area}

This study was carried out in two communities in Bayelsa state.

(a) Imringi Community (Gas Flaring Community). This community is located within Kolo Creek in Ogbia Local Government Area of Bayelsa State. Gas flaring activity has been on in this community for over 30 years. It is operated by the Shell Petroleum Development Company (SPDC). The facility has a massive horizontal flare point.

(b) Odi Community (Non-Oil Producing and Non-Gas Flaring Community).The community is located in Kolokuma/Opokuma Local Government Area of Bayelsa state.

The two communities are located in Bayelsa State which lies within, latitude $04^{0} 15^{1}$ North, $05^{0} 23^{1}$ South and longitude $05^{0} 22^{1}$ West and $06^{0} 45^{1}$ East [8]. Imiringi is the test community while Odi community served as control.

\section{Study Population}

The two communities are entirely rural communities. A total of 400 subjects were recruited for this study. This comprised:

a. 200 participants from Imiringi community (Gas flaring community)

b. 200 participants from Odi (Non-gas flaring and Non-oil producing community) as control.

Of the 200 subjects from Imiringi community, 84 were males aged between 2 and 80 years. 116 were females aged between 2 and 78 years. In Odi community, 106 were males aged between 2 and 80 years while 94 were females aged between 2 and 79 years.

\section{Advocacy, Mobilization and Pre-survey Contacts}

Ethical clearance was obtained from the Bayelsa State Ministry of Health. The traditional rulers as well as members and leaders of the community development committees of each community were met and informed about the study that was to be carried out in their various communities. Their co-operation and support were solicited in mobilizing their subjects. Meetings were held with the members of the communities and informed consent was obtained from the subjects recruited into the study.

\section{Selection Criteria}

Questionnaire was used to obtain the required information needed to include or exclude participants.

\section{Inclusion Criteria}

Subjects two years and above that consented to the study were included. The sample population was classified according to different age groups and sex.

\section{Exclusion Criteria}

Tobacco smokers and subjects with any kind of known illnesses such as cancer, diabetes mellitus and Parkinson's disease were excluded from this study. This is because cigarette smoke is an exogenous source of oxidative stress [8] while levels of oxidative stress biomarkers are known to be raised in the above mentioned disease conditions [9].

\section{Sample Collection}

Blood samples were collected by veno puncture using pyrogen free sterile disposable syringes. Samples for evaluation of oxidative stress biomarker (8-OHdG) and biochemical parameters were collected into plain serum separating tubes. They were allowed to stand for 10-20 minutes after which they were centrifuged at 3,000 rpm for 20 minutes and the serum separated using a Pasteur's pipette. Samples for measurement of haematological parameters (prothrombin time not inclusive) and heavy metals were collected into $\mathrm{K}_{3}$ EDTA anti coagulated plastic bottles and mixed thoroughly by gentle repeated turning. While samples for prothrombin time were dispensed into containers containing $3.2 \%$ tri sodium citrate at a ratio of 9 parts of blood to 1 part of $3.2 \%$ tri sodium citrate. The samples were centrifuged for 15 minutes at $3000 \mathrm{rpm}$ to obtain platelet poor plasma. The supernatant plasma was subsequently transferred into plain appendorf tubes.

\section{Laboratory Procedures}

All reagents were commercially purchased from Afro Famous Nigeria Limited, Abakpa Nike, Enugu, Enugu State and the manufacturers' SOPs were followed strictly. 


\section{International Journal of Science and Research (IJSR) \\ ISSN (Online): 2319-7064}

Index Copernicus Value (2016): 79.57 | Impact Factor (2015): 6.391

(A) 8- hydroxy -2- Deoxyguanosine (8-OHdG) [10].

The Elabscience oxidative DNA damage Elisa kit which is a competitive ELISA technique for the quantitative measurement of $8-\mathrm{OHdG}$ was used

\section{(B) Measurement of $\mathrm{Cd}, \mathrm{Se}, \mathrm{Hg}, \mathrm{Pb}$ and $\mathrm{Cr}$}

Measurement of these metals was carried out on 240 FS AA Agilent Technologies flame atomic absorption spectrometer with deuterium lamp background correction.

(C) Alanine Aminotransferase (ALT)[11]

RANDOX ALT kit was used.

(D) Aspartate Aminotransferase (AST) [11]

RANDOX AST test kit was used.

(E) Albumin [12]

RANDOX Albumin test kit was used.

(F) Bilirubin [13]

RANDOX Bilirubin kit was used.

(G) Alkaline Phosphatase (ALP) [14]

TECO Diagnostics, California, USA direct colorimetric ALP reagent kit was used.

\section{(I) Gamma-glutamyl Transferase $(\gamma$-GT) $[14]$}

RANDOX colorimetric (Kinetic Method) test kit was used.

(K) Urea [13]

RANDOX Urease-Berthelot Colorimetric method kit was used.

(L) Creatinine [15]

RANDOX Creatinine kit was used.
EA-1000B ISE electrolyte analyzer with serial number EBAAGA820/0A from Perlong Medical Equipment Company was used to measure these parameters.

(N) Platelets, Haemoglobin (Hb) [17].

SYSMEX pocH-100i automated haematology analyzer was used for the measurement of platelets in the study population.

(P) Prothrombin Time (PT) [18]

AGAPE Diagnostics Switzerland Prothrombin Time kit was used.

\section{Statistical Analysis}

Data was analyzed using Statistical Package for Social Sciences (SPSS) statistical software (Version 17 for windows) (SPSS Inc, Chicago, USA). Results were expressed as mean and standard deviation and were presented in tables.

Test of significance was done using student's t- test, Pearson correlation coefficient statistics and Tukey HSD post HOC test. Values above $95 \%$ confidence limit were considered statistically significant.

\section{Results}

Table 1 shows blood heavy metal and serum 8-OHdG concentrations in the two study groups. Highest levels of all the measured heavy metals and $8-\mathrm{OHdG}$ were observed in residents in Imiringi community as compared to Odi Community. Z-test of the means of the two groups showed statistically significant differences for all the metals and 8 OHdG $(\mathrm{p}<0.05)$.

The effect of gender induced differences on the bioaccumulation of the studied metals and $8-\mathrm{OHdG}$ in the two groups is as presented in table 2. Data show that females in Imiringi community had higher values of all the measured heavy metals and $8-\mathrm{OHdG}$ than males . The observed variations in concentrations among the two subgroups were only statistically significant in Selenium and 8-OHdG at $\mathrm{P}<0.05$ (table 2).

(M) Electrolytes $\left(\mathrm{Na}^{+}, \mathrm{K}^{+}, \mathrm{Cl}^{-}, \mathrm{HCO3}^{-}\right)[16]$

Table 1: Heavy Metal and 8-OHdG Levels in the Study Populations.

\begin{tabular}{|c|c|c|c|c|c|c|}
\hline $\begin{array}{c}\text { Study } \\
\text { Community }\end{array}$ & $\mathrm{Cd}(\mathrm{ppm})$ & $\mathrm{Cr}(\mathrm{ppm})$ & $\mathrm{Hg}(\mathrm{ppm})$ & $\mathrm{Pb}(\mathrm{ppm})$ & $\mathrm{Se}(\mathrm{ppm})$ & $8-\mathrm{OHdG}$ \\
\hline $\begin{array}{c}\text { Imiringi } \\
(\mathrm{n}=200)\end{array}$ & $0.030 \pm 0.006$ & $0.026 \pm 0.009$ & $0.829 \pm 0.206$ & $10.794 \pm 1.513$ & $2.418 \pm 0.893$ & $63.85 \pm 34.15$ \\
\hline $\begin{array}{c}\text { Odi } \\
(\mathrm{n}=200)\end{array}$ & $0.017 \pm 0.01$ & $0.004 \pm 0.011$ & $0.081 \pm 0.098$ & $0.929 \pm 0.314$ & $0.037 \pm 0.064$ & $25.17 \pm 12.16$ \\
\hline P Value & $<0.0001(\mathrm{~S})$ & $<0.0001(\mathrm{~S})$ & $<0.0001(\mathrm{~S})$ & $<0.0001(\mathrm{~S})$ & $<0.0001(\mathrm{~S})$ & $<0.0001(\mathrm{~S})$ \\
\hline
\end{tabular}

$\mathrm{S}=$ Significant

Results from Odi community show that females had higher mean values of Cadmium, Chromium, Lead and Selenium while males had higher mean values of Mercury and $8-\mathrm{OHdG}$ than females. These observed differences were significant in all the measured metals except in Cadmium and Lead at $\mathrm{P}<0.05$ (table 2). 
International Journal of Science and Research (IJSR)

ISSN (Online): 2319-7064

Index Copernicus Value (2016): 79.57 | Impact Factor (2015): 6.391

Table 2: Heavy Metal and 8-OHdG Levels in Males and Females

\begin{tabular}{|c|c|c|c|c|c|c|c|c|}
\hline \multicolumn{2}{|c|}{ Imiringi } & \multicolumn{3}{c|}{ Odi } \\
\hline \multicolumn{2}{|c|}{$\begin{array}{l}\text { Males } \\
\mathrm{n}=84\end{array}$} & $\begin{array}{c}\text { Females } \\
\mathrm{n}=116\end{array}$ & F value & P value & $\begin{array}{c}\text { Males } \\
\mathrm{n}=106\end{array}$ & $\begin{array}{c}\text { Females } \\
\mathrm{n}=94\end{array}$ & F value & P value \\
\hline $\mathrm{Cd}(\mathrm{ppm})$ & $0.031 \pm 0.007$ & 1.119 & 1.119 & 0.292 & $0.017 \pm 0.014$ & $0.018 \pm 0.006$ & 0.412 & 0.522 \\
\hline $\mathrm{Cr}(\mathrm{ppm})$ & $0.026 \pm 0.010$ & 0.487 & 0.487 & 0.486 & $0.04 \pm 0.013$ & $0.004 \pm 0.009$ & 505.75 & $<0.0001(\mathrm{~S})$ \\
\hline $\mathrm{Hg}(\mathrm{ppm})$ & $0.84 \pm 0.225$ & 0.579 & 0.579 & 0.448 & $0.109 \pm 0.122$ & $0.052 \pm 0.06$ & 16.89 & $<0.0001(\mathrm{~S})$ \\
\hline $\mathrm{Pb}(\mathrm{ppm})$ & $10.91 \pm 1.36$ & 1.580 & 1.580 & 0.210 & $0.732 \pm 0.212$ & $0.741 \pm 0.18$ & 0.103 & 0.748 \\
\hline $\begin{array}{c}8-\mathrm{OHdG} \\
(\mathrm{pg} / \mathrm{ml})\end{array}$ & $54.0 \pm 20.47$ & 63.2538 .09 & 4.094 & $0.044(\mathrm{~S})$ & $28.13 \pm 23.89$ & $23.30 \pm 27.89$ & 1.742 & 0.1884 \\
\hline
\end{tabular}

$\mathrm{S}=$ Significant

The mean concentrations of the measured heavy metals and $8-\mathrm{OHdG}$ in the different age groups of Imiringi community are as tabulated in table 3 . Test of significance of these observed age induced differences using Tukey post HOC analysis are presented in table 4 . The highest level of Cadmium was observed in residents 51- 60 years old $(0.034 \pm 0.005 \mathrm{ppm}$; Range: $0.027-0.041)$. The least mean concentration of Cadmium $(0.020 \pm 0.004 \mathrm{ppm}$; Range: $0.02-0.028$ ) was observed in residents $2-10$ years old. These values were statistically significantly different at $\mathrm{P}<0.05$ (Table 3). The highest mean value of chromium (0.032 \pm 0.013 ppm; Range: $0.015-0.048)$ was recorded in the age group 31-40 years while the least value $(0.020 \pm 0.003 \mathrm{ppm}$; Range: $0.01-0.034)$ was recorded among residents $2-10$ years. This difference was not statistically significant. Subjects $71-80$ years old, had the highest mean concentrations of mercury $(0.971 \pm$ 0.084ppm; Range: 0.843 - 1.064), Lead (11.85 \pm 1.47ppm; Range: 9.41- 13.17) and Selenium (3.14 \pm 1.32ppm; Range: $1.24-4.78)$. The least mean values of Mercury (0.436 $\pm 0.142 p p m$; Range: $0.427-0.677)$, Lead $(9.14 \pm 0.81 \mathrm{ppm}$; Range: $8.242-10.08)$ and Selenium (1.842 $\pm 0.31 \mathrm{ppm}$; Range: $1.24-2.06)$ were measured among residents between the ages of $2-10$ years old. These observed differences were statistically significant at $\mathrm{P}<0.05$ (Table 4). It also shows that residents within the ages of $51-60$ years had the highest mean concentration of 8 -OHdG $(82.40 \pm 22.24 \mathrm{pg} / \mathrm{ml})$ while least levels of 8 OHdG was observed in residents between the ages of $31-$ 40 years. These observed differences were statistically significant at $\mathrm{P}<0.05$.

Table 3: Heavy Metals and 8-OHdG Levels in Subjects in Relation to Age in Imiringi Community.

\begin{tabular}{|l|l|l|l|l|c|c|}
\hline Age Range & \multicolumn{1}{|c|}{$\mathrm{Cd}(\mathrm{ppm})$} & $\mathrm{Cr}(\mathrm{ppm}$ & $\mathrm{Hg}(\mathrm{ppm})$ & $\mathrm{Pb}(\mathrm{ppm})$ & $\mathrm{Se}(\mathrm{ppm})$ & $8-\mathrm{OHdG}(\mathrm{pg} / \mathrm{ml})$ \\
\hline $2-10(\mathrm{n}=30)$ & $0.020 \pm 0.004$ & $0.020 \pm 0.003$ & $0.436 \pm 0.142$ & $9.14 \pm 0.81$ & $1.842 \pm 0.31$ & $42.15 \pm 34.75$ \\
\hline $11-20(\mathrm{n}=34)$ & $0.025 \pm 0.006$ & $0.022 \pm 0.08$ & $0.835 \pm 0.12$ & $10.87 \pm 1.82$ & $2.043 \pm 0.37$ & $59.00 \pm 36.60$ \\
\hline $21-30(\mathrm{n}=26)$ & $0.028 \pm 0.004$ & $0.027 \pm 0.006$ & $0.803 \pm 0.14$ & $10.654 \pm 1.31$ & $2.054 \pm 0.42$ & $42.40 \pm 16.60$ \\
\hline $31-40(\mathrm{n}=30)$ & $0.029 \pm 0.005$ & $0.032 \pm 0.013$ & $0.820 \pm 0.21$ & $10.72 \pm 0.85$ & $2.098 \pm 0.42$ & $37.60 \pm 15.04$ \\
\hline $41-50(\mathrm{n}=23)$ & $0.030 \pm 0.005$ & $0.028 \pm 0.009$ & $0.685 \pm 0.22$ & $9.864 \pm 0.77$ & $2.20 \pm 0.50$ & $58.40 \pm 18.24$ \\
\hline $51-60(\mathrm{n}=25)$ & $0.034 \pm 0.005$ & $0.026 \pm 0.01$ & $0.696 \pm 0.23$ & $10.24 \pm 0.65$ & $2.50 \pm 0.87$ & $82.40 \pm 22.24$ \\
\hline $61-70(\mathrm{n}=15)$ & $0.032 \pm 0.005$ & $0.025 \pm 0.008$ & $0.74 \pm 0.09$ & $10.87 \pm 0.55$ & $2.94 \pm 0.78$ & $80.10 \pm 20.31$ \\
\hline $71-80(\mathrm{n}=17)$ & $0.03 \pm 0.01$ & $0.024 \pm 0.01$ & $0.971 \pm 0.08$ & $11.85 \pm 1.47$ & $3.14 \pm 1.32$ & $76.40 \pm 35.26$ \\
\hline
\end{tabular}

The mean concentrations of the measured heavy metals and $8-\mathrm{OHdG}$ in the different age groups of Odi community are as tabulated in table 5 . Test of significance of these observed age induced differences are presented in table 6.The highest mean value of Cadmium (0.022 \pm 0.01ppm; Range: $0.009-0.055)$, Mercury (0.097 \pm 0.08 ; Range: $0.0-0.222$ and Selenium $(0.098 \pm 0.08 \mathrm{ppm}$; Range: $0.0-0.188$ ) were recorded in residents between the ages of $71-80$ years. Highest mean values of Chromium (0.038 \pm 0.07 ppm; Range: $0.0-0.168)$ and Lead (0.919 \pm 0.25ppm; Range: 0.494 - 0.934) were recorded in residents $11-20$ and $31-40$ years respectively. Children $2-10$ years old had the least mean values of Cadmium (0.012 $\pm 0.008 p p m$; Range: $0.009-$ $0.025)$, Lead (0.502 $\pm 0.101 \mathrm{ppm}$; Range: $0.494-0.811)$ and Selenium $(0.045 \pm 0.024$; Range $0.036-0.078)$. Least mean values of Chromium (0.001 \pm 0.001 ; Range: $0.0-$ $0.024)$ and Mercury (0.001 \pm 0.001 ; Range: $0.00-0.004)$ were observed in residents $71-80,21-30$ and $11-20$ years respectively. The observed differences in these age groups were statistically different at $\mathrm{P}<0.05$. The highest mean concentration of $8-\mathrm{OHdG}(52.6 \pm 11.59 \mathrm{pg} / \mathrm{ml}$;
Range: 40 - 73) was recorded in residents 71 -- 80 years old while residents that were between 2-10 years old recorded the least mean values. These measured variations in 8 -OHdG induced by differences in age were significantly different at $\mathrm{P}<0.05$. The Correlation between measured metals and $8-\mathrm{OHdG}$ in Imiringi community is presented in table $7.8-\mathrm{OHdG}$ correlated weakly but positively with Lead $(\mathrm{r}=0.339)$ and Chromium $(\mathrm{r}=$ 0.238). These observed correlations were statistically significant at $\mathrm{P}<0.005$. There was no significant correlation between $8-\mathrm{OHdG}$ and the other measured heavy metals.

Table 8 is a representation of the mean values of measured liver function parameters in the study groups. It shows that residents in Imiringi community had the highest mean values of all the measured liver function parameters with the exception of albumin and total protein which were highest in the residents of the control community.

Results of kidney function parameters measured in the study populations are as presented in table 9 


\section{International Journal of Science and Research (IJSR) \\ ISSN (Online): 2319-7064}

Index Copernicus Value (2016): 79.57 | Impact Factor (2015): 6.391

Table 4: Comparison of Heavy Metals and 8-OHdG Levels in Subjects in Relation to Age in Imiringi Community.

\begin{tabular}{|c|c|c|c|c|c|c|}
\hline Age Range & Cd (P Value) & Cr (P Value) & Hg (PValue) & $\mathrm{Pb}(\mathrm{P}$ Value $)$ & Se (P Value) & 8-OHdG (P Value) \\
\hline $2-10$ vs $11-20$ & 0.7391 & 1.0000 & $<0.0001(\mathrm{~S})$ & $<0.001(\mathrm{~S})$ & 0.9095 & 0.1961 \\
\hline $2-10$ vs $21-30$ & $<0.001(\mathrm{~S})$ & 0.9946 & $<0.0001(\mathrm{~S})$ & $<0.001(\mathrm{~S})$ & 0.9155 & 1.0000 \\
\hline $2-10$ vs $31-40$ & $<0.001(\mathrm{~S})$ & 0.8737 & $<0.0001(\mathrm{~S})$ & $<0.001(\mathrm{~S})$ & 0.7695 & 0.9979 \\
\hline $2-10$ vs $41-50$ & $<0.001(\mathrm{~S})$ & 0.9902 & $<0.0001(\mathrm{~S})$ & 0.3155 & 0.4571 & 0.3620 \\
\hline $2-10$ vs $51-60$ & $<0.001(\mathrm{~S})$ & 0.9981 & $<0.0001(\mathrm{~S})$ & $0.0119(\mathrm{~S})$ & $0.0041(\mathrm{~S})$ & $<0.001(\mathrm{~S})$ \\
\hline $2-10$ vs $61-70$ & $0.0334(\mathrm{~S})$ & 0.9998 & $<0.0001(\mathrm{~S})$ & $<0.001(\mathrm{~S})$ & $<0.001(\mathrm{~S})$ & $0.0003(\mathrm{~S})$ \\
\hline $2-10$ vs $71-80$ & 0.1384 & 0.9999 & $<0.0001(\mathrm{~S})$ & $<0.0001(\mathrm{~S})$ & $<0.0001(\mathrm{~S})$ & $0.0010(\mathrm{~S})$ \\
\hline $11-20$ vs $21-30$ & $0.0034(\mathrm{~S})$ & 0.9992 & 0.9959 & 0.9958 & 1.0000 & 0.2569 \\
\hline $11-20$ vs $31-40$ & $<0.001(\mathrm{~S})$ & 0.9397 & 1.0000 & 0.9995 & 1.0000 & $0.0345(\mathrm{~S})$ \\
\hline $11-20$ vs $41-50$ & $<0.001(\mathrm{~S})$ & 0.9981 & $0.0237(\mathrm{~S})$ & $0.0301(\mathrm{~S})$ & 0.9839 & 1.0000 \\
\hline $11-20$ vs $51-60$ & 0.0483 & 0.9998 & $0.0391(\mathrm{~S})$ & 0.4328 & 0.1168 & $0.0235(\mathrm{~S})$ \\
\hline $11-20$ vs $61-70$ & 0.5110 & 1.0000 & 0.6007 & 1.0000 & $0.0002(\mathrm{~S})$ & 0.1839 \\
\hline $11-20$ vs $71-80$ & 0.8723 & 1.0000 & 0.1190 & 0.0851 & $<0.001(\mathrm{~S})$ & 0.3633 \\
\hline $21-30$ vs $31-40$ & $0.005(\mathrm{~S})$ & 0.9994 & 0.9999 & 1.0000 & 1.0000 & 0.9977 \\
\hline $21-30$ vs $41-50$ & 0.9967 & 1.0000 & 0.2188 & 0.2540 & 0.9926 & 0.4262 \\
\hline $21-30$ vs $51-60$ & 0.9962 & 1.0000 & 0.3099 & 0.9081 & 0.1950 & $<0.0001(\mathrm{~S})$ \\
\hline $21-30$ vs $61-70$ & 0.9157 & 1.0000 & 0.9421 & 0.9990 & $0.0007(\mathrm{~S})$ & $0.0006(\mathrm{~S})$ \\
\hline $21-30$ vs $71-80$ & 0.5193 & 1.0000 & $0.0322(\mathrm{~S})$ & $0.0218(\mathrm{~S})$ & $<0.001(\mathrm{~S})$ & $0.0017(\mathrm{~S})$ \\
\hline $31-40$ vs $41-50$ & 0.0733 & 0.9999 & 0.0766 & 0.1337 & 0.9991 & 0.1000 \\
\hline $31-40$ vs $51-60$ & $0.0003(\mathrm{~S})$ & 0.9981 & 0.1189 & 0.7845 & 0.2747 & $<0.0001(\mathrm{~S})$ \\
\hline $31-40$ vs $61-70$ & $0.0003(\mathrm{~S})$ & 0.9981 & 0.8012 & 0.9999 & $<0.0001(\mathrm{~S})$ & $<0.0001(\mathrm{~S})$ \\
\hline $31-40$ vs $71-80$ & $<0.0001(\mathrm{~S})$ & 0.9943 & 0.0650 & $0.0304(\mathrm{~S})$ & $<0.0001(\mathrm{~S})$ & $<0.0001(\mathrm{~S})$ \\
\hline $41-50$ vs $51-60$ & 0.8553 & 1.0000 & 1.0000 & 0.9492 & 0.7249 & $0.0447(\mathrm{~S})$ \\
\hline $41-50$ vs $61-70$ & 0.5995 & 1.0000 & 0.9755 & 0.1501 & $0.0122(\mathrm{~S})$ & 0.2270 \\
\hline $41-50$ vs $71-80$ & 0.1877 & 1.0000 & $<0.0001(\mathrm{~S})$ & $<0.0001(\mathrm{~S})$ & $0.0002(\mathrm{~S})$ & 0.4169 \\
\hline $51-60$ vs $61-70$ & 0.9986 & 1.0000 & 0.9927 & 0.7024 & 0.4003 & 1.0000 \\
\hline $51-60$ vs $71-80$ & 0.9020 & 1.0000 & $<0.0001(\mathrm{~S})$ & $0.0004(\mathrm{~S})$ & $0.0321(\mathrm{~S})$ & 0.9965 \\
\hline $61-70$ vs $71-80$ & 0.9992 & 1.0000 & $0.0033(\mathrm{~S})$ & 0.2452 & 0.9865 & 0.9999 \\
\hline
\end{tabular}

The table shows that highest levels of sodium $(141 \pm$ $2.2 \mathrm{mmol} / \mathrm{l})$, chloride $(103 \pm 3.6)$ and bicarbonate $(29.8 \pm$ 4.4) were recorded in residents in Odi. potassium $(4.4 \pm$ $0.4 \mathrm{mmol} / \mathrm{l})$, creatinine $(101 \pm 4.0 \mu \mathrm{mol} / \mathrm{l})$ and urea $(6.6 \pm$
$0.9 \mathrm{mmol} / \mathrm{l})$. These differences were statistically significant at $\mathrm{p}<0.05$

Residents in Imiringi had the highest levels of

Table 5: Metal Levels and 8-OHdG Levelsin Subjects in Relation to Age in Odi Community.

\begin{tabular}{|c|c|c|c|c|c|c|}
\hline Age Range & $\mathrm{Cd}(\mathrm{ppm})$ & $\mathrm{Cr}(\mathrm{ppm}$ & $\mathrm{Hg}(\mathrm{ppm})$ & $\mathrm{Pb}(\mathrm{ppm})$ & $\mathrm{Se}(\mathrm{ppm})$ & $8-\mathrm{OHdG}(\mathrm{ng} / \mathrm{ml})$ \\
\hline $2-10(\mathrm{n}=27)$ & $0.012 \pm 0.008$ & $0.021 \pm 0.004$ & $0.016 \pm 0.014$ & $0.502 \pm 0.101$ & $0.045 \pm 0.024$ & $27.70 \pm 11.22$ \\
\hline $11-20(\mathrm{n}=36)$ & $0.018 \pm 0.004$ & $0.038 \pm 0.07$ & $0.021 \pm 0.02$ & $0.612 \pm 0.16$ & $0.081 \pm 0.011$ & $30.80 \pm 9.96$ \\
\hline $21-30(\mathrm{n}=26)$ & $0.016 \pm 0.004$ & $0.010 \pm 0.002$ & $0.001 \pm 0.001$ & $0.666 \pm 0.17$ & $0.056 \pm 0.063$ & $33.50 \pm 10.06$ \\
\hline $31-40(\mathrm{n}=32)$ & $0.013 \pm 0.003$ & $0.012 \pm 0.002$ & $0.002 \pm 0.002$ & $0.919 \pm 0.25$ & $0.055 \pm 0.05$ & $30.80 \pm 10.06$ \\
\hline $41-50(\mathrm{n}=20)$ & $0.014 \pm 0.002$ & $0.009 \pm 0.001$ & $0.06 \pm 0.04$ & $0.900 \pm 0.09$ & $0.057 \pm 0.004$ & $38.40 \pm 11.06$ \\
\hline $51-60(\mathrm{n}=21)$ & $0.016 \pm 0.02$ & $0.006 \pm 0.001$ & $0.06 \pm 0.04$ & $0.90 \pm 0.09$ & $0.060 \pm 0.04$ & $43.80 \pm 17.06$ \\
\hline $61-70(\mathrm{n}=20)$ & $0.017 \pm 0.01$ & $0.004 \pm 0.001$ & $0.07 \pm 0.08$ & $0.864 \pm 0.08$ & $0.065 \pm 0.07$ & $48.30 \pm 12.06$ \\
\hline $71-80(\mathrm{n}=18)$ & $0.022 \pm 0.01$ & $0.001 \pm 0.001$ & $0.097 \pm 0.08$ & $0.818 \pm 0.08$ & $0.098 \pm 0.08$ & $52.67 \pm 13.59$ \\
\hline
\end{tabular}

Table 10 shows the mean levels of the different measured haematological parameters in the study communities. There was statistically significant lower levels of $\mathrm{Hb}$, WBC and Platelet in the test community (Imiringi) $(p<0.05)$ compared to the control community (Odi). Subjects in Odi community recorded the highest levels of $\mathrm{Hb}(12.02 \pm 1.53 \mathrm{~g} / \mathrm{dl}), \operatorname{WBC}\left(6.6 \pm 0.63 \times 10^{9} / 1\right)$ and Platelets $\left(208.5 \pm 7.52 \times 10^{9} / 1\right)$.
In contrast, the length of bleeding time was prolonged in the residents of Imiringi community $(13.87 \pm 1.14$ seconds) than in the subjects from Odi community $(12.74$ \pm 1.09 ). These differences in prothrombin time in the two populations were statistically significant. 


\section{International Journal of Science and Research (IJSR) \\ ISSN (Online): 2319-7064}

Index Copernicus Value (2016): 79.57 | Impact Factor (2015): 6.391

Table 6: Comparison of Heavy Metals and 8-OHdG Levels in Different Age Groups in Odi Community

\begin{tabular}{|c|c|c|c|c|c|c|}
\hline Age Range & Cd (P Value) & $\mathrm{Cr}$ (P Value) & $\mathrm{Hg}$ (P Value) & $\mathrm{Pb}$ (P Value) & Se(P Value) & $\begin{array}{c}\text { 8OHdG (P } \\
\text { Value) }\end{array}$ \\
\hline $2-10$ vs $11-20$ & 0.1445 & 0.2479 & 0.9999 & 0.0567 & 0.1371 & 0.9681 \\
\hline $2-10$ vs $21-30$ & 0.7280 & 0.8390 & 0.9458 & $0.0011(\mathrm{~S})$ & 0.9950 & 0.6222 \\
\hline $2-10$ vs $31-40$ & 0.9999 & 0.9202 & 0.9509 & $<0.0001(\mathrm{~S})$ & 0.9962 & 0.9724 \\
\hline $2-10$ vs $41-50$ & 0.9948 & 0.8275 & $0.0408(\mathrm{~S})$ & $<0.0001(\mathrm{~S})$ & 0.9945 & $0.0466(\mathrm{~S})$ \\
\hline $2-10$ vs $51-60$ & 0.7821 & 0.5861 & $0.0359(\mathrm{~S})$ & $<0.0001(\mathrm{~S})$ & 0.9776 & $0.0001(\mathrm{~S})$ \\
\hline $2-10$ vs $61-70$ & 0.5493 & 0.4385 & $0.0039(\mathrm{~S})$ & $<0.0001(\mathrm{~S})$ & 0.9052 & $0.0001(\mathrm{~S})$ \\
\hline $2-10$ vs $71-80$ & $0.0001(\mathrm{~S})$ & 0.0616 & $<0.0001(\mathrm{~S})$ & $<0.0001(\mathrm{~S})$ & $.0011(\mathrm{~S})$ & $0.0001(\mathrm{~S})$ \\
\hline $11-20$ vs $21-30$ & 0.9881 & 0.0031 & 0.7311 & 0.8256 & 0.5970 & 0.9863 \\
\hline $11-20$ vs $31-40$ & 0.2914 & 0.0039 & 0.7247 & $<0.0001(\mathrm{~S})$ & 0.4698 & 1.0000 \\
\hline $11-20$ vs $41-50$ & 0.7428 & 0.0058 & 0.0706 & $<0.0001(\mathrm{~S})$ & 0.7344 & 0.2875 \\
\hline $11-20$ vs $51-60$ & 0.9919 & 0.0011 & 0.0623 & $<0.0001(\mathrm{~S})$ & 0.8353 & $0.0020(\mathrm{~S})$ \\
\hline $11-20$ vs $61-70$ & 0.9999 & 0.0005 & $0.0068(\mathrm{~S})$ & $<0.0001(\mathrm{~S})$ & 0.9596 & $0.0001(\mathrm{~S})$ \\
\hline $11-20$ vs $71-80$ & 0.4583 & $.0001(\mathrm{~S})$ & $<0.0001(\mathrm{~S})$ & $<0.0001(\mathrm{~S})$ & 0.8294 & $0.0001(\mathrm{~S})$ \\
\hline $21-30$ vs $31-40$ & 0.9066 & 1.0000 & 1.0000 & $<0.0001(\mathrm{~S})$ & 1.0000 & 0.9882 \\
\hline $21-30$ vs $41-50$ & 0.9950 & 1.0000 & $0.0011(\mathrm{~S})$ & $<0.0001(\mathrm{~S})$ & 1.0000 & 0.8549 \\
\hline $21-30$ vs $51-60$ & 1.0000 & 0.9997 & $0.0009(\mathrm{~S})$ & $<0.0001(\mathrm{~S})$ & 1.0000 & 0.0614 \\
\hline $21-30$ vs $61-70$ & 0.9999 & 0.9962 & $<0.0001(\mathrm{~S})$ & $0.0002(\mathrm{~S})$ & 0.9992 & $0.0009(\mathrm{~S})$ \\
\hline $21-30$ vs $71-80$ & 0.1082 & 0.8878 & $<0.0001(\mathrm{~S})$ & $0.0005(\mathrm{~S})$ & $.0278(\mathrm{~S})$ & $0.0001(\mathrm{~S})$ \\
\hline $31-40$ vs $41-50$ & 0.9999 & 0.9999 & $0.0007(\mathrm{~S})$ & 0.9998 & 1.0000 & 0.3150 \\
\hline $31-40$ vs $51-60$ & 0.9311 & 0.9946 & $0.0006(\mathrm{~S})$ & 0.9998 & 1.0000 & $0.0028(\mathrm{~S})$ \\
\hline $31-40$ vs $61-70$ & 0.7636 & 0.9729 & $<0.0001(\mathrm{~S})$ & 0.8799 & 0.9978 & $0.0001(\mathrm{~S})$ \\
\hline $31-40$ vs $71-80$ & $0.0004(\mathrm{~S})$ & 0.6674 & $<0.0001(\mathrm{~S})$ & $0.0461(\mathrm{~S})$ & $0.0105(\mathrm{~S}$ & $0.0001(\mathrm{~S})$ \\
\hline $41-50$ vs $51-60$ & 0.9963 & 1.0000 & 1.0000 & 1.0000 & 1.0000 & 0.8213 \\
\hline $41-50$ vs $61-70$ & 0.9630 & 0.9992 & 0.9978 & 0.9933 & 0.9997 & 0.1394 \\
\hline $41-50$ vs $71-80$ & $0.0191(\mathrm{~S})$ & 0.9605 & 0.0739 & 0.3865 & 0.0754 & $0.0058(\mathrm{~S})$ \\
\hline $51-60$ vs $61-70$ & 1.0000 & 1.0000 & 0.9976 & 0.9927 & 1.0000 & 0.9230 \\
\hline $51-60$ vs $71-80$ & 0.1699 & 0.9973 & 0.0645 & 0.3637 & 0.1152 & 0.2713 \\
\hline $61-70$ vs $71-80$ & 0.4110 & 0.9999 & 0.3996 & 0.9295 & 0.2757 & 0.9457 \\
\hline
\end{tabular}

Table 7: Correlation between Measured Metals and 8-OHdG in Imiringi Community

\begin{tabular}{|c|c|c|c|c|c|c|c|c|c|c|}
\hline $\begin{array}{c}8- \\
\text { OHdG }\end{array}$ & \multicolumn{2}{|c|}{ Cadmium } & \multicolumn{2}{c|}{ Chromium } & \multicolumn{2}{c|}{ Mercury } & \multicolumn{2}{c|}{ Pb(ppm) } & \multicolumn{2}{c|}{ Se(ppm) } \\
\cline { 2 - 11 } & $\mathrm{R}$ & $\mathrm{P}$ & $\mathrm{R}$ & $\mathrm{p}$ & $\mathrm{R}$ & $\mathrm{P}$ & $\mathrm{R}$ & $\mathrm{P}$ & $\mathrm{R}$ & $\mathrm{P}$ \\
\hline & 0.060 & 0.399 & 0238 & $0.001(\mathrm{~S})$ & 0.023 & 0.747 & 0.339 & $<0.001(\mathrm{~S})$ & 0.066 & 0.353 \\
\hline
\end{tabular}

Table 8: Mean and SD Values of Liver Function Parameters in the Study Populations

\begin{tabular}{|c|c|c|c|c|c|c|c|c|}
\hline $\begin{array}{c}\text { STUDY } \\
\text { COMMUNITY } \\
\mathrm{n}=200\end{array}$ & $\begin{array}{c}\text { ALT } \\
(\mathrm{U} / \mathrm{I})\end{array}$ & $\begin{array}{c}\text { AST } \\
(\mathrm{U} / \mathrm{I})\end{array}$ & $\begin{array}{c}\text { G-GT } \\
(\mathrm{U} / \mathrm{I})\end{array}$ & $\begin{array}{c}\text { ALP } \\
(\mathrm{U} / \mathrm{I})\end{array}$ & $\begin{array}{c}\text { Albumin } \\
(\mathrm{g} / \mathrm{l})\end{array}$ & $\begin{array}{c}\text { TP } \\
(\mathrm{g} / \mathrm{l})\end{array}$ & $\begin{array}{c}\text { TB } \\
(\mu \mathrm{mol} / \mathrm{l})\end{array}$ & $\begin{array}{c}\mathrm{CB} \\
(\mu \mathrm{mol} / \mathrm{l})\end{array}$ \\
\hline Imiringi & $7.2 \pm 1.6$ & $10.5 \pm 2$ & $40.4 \pm 3.3$ & $24 \pm 2$ & $37.4 \pm 3$ & $63 \pm 1$ & $9 \pm 0.88$ & $2.8 \pm 0.24$ \\
\hline Odi & $5.6 \pm 1.3$ & $9.5 \pm 1.8$ & $25.5 \pm 4.2$ & $22.3 \pm 1$ & $38.5 \pm 2$ & $63.8 \pm 1.3$ & $5.7 \pm 0.1$ & $2.0 \pm 0.11$ \\
\hline P Value & $<0.0001(\mathrm{~S})$ & $<0.0001(\mathrm{~S})$ & $<0.001(\mathrm{~S})$ & $<0.0001(\mathrm{~S})$ & $<0.0001(\mathrm{~S})$ & $<0.0001(\mathrm{~S})$ & $<0.0001(\mathrm{~S})$ & $<0.001(\mathrm{~S})$ \\
\hline
\end{tabular}

Table 9: Mean and SD Values of Kidney Function Parameters in the Study Populations.

\begin{tabular}{|c|c|c|c|c|c|c|}
\hline $\begin{array}{c}\text { Study } \\
\text { Community }\end{array}$ & $\begin{array}{c}\mathrm{Na}^{+} \\
(\mathrm{mmol} / \mathrm{l})\end{array}$ & $\begin{array}{c}\mathrm{K}^{+} \\
(\mathrm{mmol} / \mathrm{l})\end{array}$ & $\begin{array}{c}\mathrm{Cl}^{-} \\
(\mathrm{mmol} / \mathrm{l})\end{array}$ & $\begin{array}{c}\mathrm{HCO}_{3}^{-} \\
(\mathrm{mm} 0 \mathrm{l} / \mathrm{l})\end{array}$ & $\begin{array}{c}\text { Creatinine } \\
(\mu \mathrm{mol} / \mathrm{l})\end{array}$ & $\begin{array}{c}\text { Urea } \\
(\mathrm{mmol} / \mathrm{l})\end{array}$ \\
\hline Imiringi & $139 \pm 3.3$ & $4.4 \pm 0.4$ & $100 \pm 3.2$ & $25.4 \pm 3.0$ & $101 \pm 4.0$ & $6.6 \pm 0.9$ \\
\hline Odi & $141 \pm 2.2$ & $4.0 \pm 0.5$ & $103 \pm 3.6$ & $29.8 \pm 4.4$ & $97.7 \pm 4.0$ & $6.0 \pm 0.7$ \\
\hline P Value & $<0.0001(\mathrm{~S})$ & $<0.0001(\mathrm{~S})$ & $<0.0001(\mathrm{~S})$ & $<0.0001(\mathrm{~S})$ & $<0.0001(\mathrm{~S})$ & $<0.0001(\mathrm{~S})$ \\
\hline
\end{tabular}

$\mathrm{S}=$ Significant

Table 10: Mean and SD Values of Haematological Parameters in the Study Populations.

\begin{tabular}{|c|c|c|c|c|}
\hline STUDY COMMUNITY & $\mathrm{Hb}(\mathrm{g} / \mathrm{dl})$ & WBC $\left(\times 10^{9} / \mathrm{l}\right)$ & Platelet $\left(\times 10^{9} / \mathrm{l}\right)$ & PT (Seconds) \\
\hline Imiringi & $11.3 \pm 1.9$ & $5.8 \pm 0.55$ & $186.3 \pm 6.3$ & $13.87 \pm 1.14$ \\
\hline Odi & $12.02 \pm 1.5$ & $6.6 \pm 0.63$ & $208.5 \pm 7.5$ & $12.74 \pm 1.1$ \\
\hline P Value & $<0.0001(\mathrm{~S})$ & $<0.0001(\mathrm{~S})$ & $<0.0001(\mathrm{~S})$ & $<0.001(\mathrm{~S})$ \\
\hline
\end{tabular}

$\mathrm{S}=$ Significant

Volume 6 Issue 12, December 2017

www.ijsr.net

Licensed Under Creative Commons Attribution CC BY 


\section{International Journal of Science and Research (IJSR) \\ ISSN (Online): 2319-7064}

Index Copernicus Value (2016): 79.57 | Impact Factor (2015): 6.391

\section{Discussion}

The results of this study have shown high levels of bioaccumulation of metals in the blood samples of residents in the gas flaring community in relation to the control community. The differences in the levels of these metals in the blood samples of these residents were equally found to be statistically significant $(\mathrm{P}<0.05)$. All the measured values of these metals in the two communities were above the recommended reference ranges except for selenium which was found to be within the reference range in the control community. These findings, point to widespread pollution which varies significantly depending on the predominant activities in the area. The highest levels of Cadmium, Chromium, Mercury, Lead and Selenium found in the blood samples of residents in gas flaring community could be attributed to the fact that inhalation of oxides of these metals may result in increased metal uptake [19]. Oxides of these metals are released in large amounts into the atmosphere continually through the process of gas flaring. These oxides mix with rain and are equally deposited on vegetation, and water bodies in the community which are in turn consumed by the residents [20] [21]. Up to 10 $40 \%$ retention of inhaled Cadmium in the human body had been reported [19].

There was no clear cut pattern in the level of these measured metals in males and females. Though females in the gas flaring community (Imiringi) exhibited the highest levels of all the metals, statistically significant difference was only observed in selenium. Some authors also reported significantly higher blood levels in males than in females for lead and cadmium [22], cadmium [23], lead [24], and nickel [25]. Conversely, some other authors [26] and [23] reported no difference between heavy metals blood concentrations in men and women. An assessment of the effect of age differences on heavy metal bioaccumulation in the subjects shows different patterns of accumulation for different metals in different age groups. In the gas flaring community and the control community, it was observed that bioaccumulation of measured metals increased with age.

In this study, the serum levels of the oxidative stress biomarker and indicator of DNA oxidative damage (8$\mathrm{OHdG}$ ) was higher in Imiringi than in the control community just as observed in the measured metals. The results suggest that the metals influenced the concentrations of the oxidative stress biomarker as can be seen in the positive correlations between them. This suggests strongly that lead and chromium are strong oxidative factors eliciting high levels of $8-\mathrm{OHdG}$ which is an indicator of oxidative DNA damage. Lead affects the red blood cells and the haematopoietic system, the immune system as well as causes damage to organs such as the liver, kidneys, heart and male gonads [27]. Serum $8-\mathrm{OHdG}$ was found to be significantly higher in patients with colorectal cancer compared with patients without colorectal cancer [28]. Exposure to Cadmium can cause a variety of pathological alterations in several organs and tissues as well as induce diabetic complications, hypertension and osteoporosis [29]. Lead, cadmium and mercury deplete cells' major anti-oxidants and enzymes. Either redox-active or redox-inactive metals may cause an increase in production of reactive oxygen species such as the hydroxyl radical (HO-) superoxide radical $\left(\mathrm{O}_{2}^{-}\right)$or hydrogen peroxide $\left(\mathrm{H}_{2} \mathrm{O}_{2}\right)$. Enhanced generation of reactive oxygen species can overwhelm cells' intrinsic antioxidant defences and result in oxidative stress. The results of this study suggest a higher possibility of occurrence of renal impairment in the residents of the gas flaring community. This assertion is supported by the statistically significantly increased levels of serum urea and serum creatinine found in these two communities compared to those in the control group. This is in line with the findings by Egwurugwu et al [21] who also reported statistically significantly increased serum concentrations of urea, creatinine, potassium, uric acid and inorganic phosphate in subjects exposed to oil and gas flares.

Exposure to gas flares is known to cause some adverse changes in haematological parameters. These changes affect blood and blood-forming cells negatively and could give rise to anaemia (aplastic), pancytopenia and leukaemia [30]. The results of the measured haematological indices equally show that the residents in the gas flaring community may be prone to anaemia due to significantly lower levels of haemoglobin measured among them. Residents in Imiringi community may also be prone to suppressed immunity as well as bleeding disorders as evidenced by the significantly lower levels of WBC, platelets and longer prothrombin time measured in their blood samples as compared to the control.

\section{Conclusion}

The findings of this study reveal bioaccumulation of Cadmium, Chromium, Lead, Mercury and Selenium in blood samples of subjects in Imiringi community. Higher levels of the oxidative DNA damage biomarker: 8Hydroxy-2-deoxyguanosine was equally observed.

The following measured biochemical indices: AST, ALT, ALP, Gamma-GT, total bilirubin, conjugated bilirubin potassium and creatinine were found to be elevated in subjects in the test community.

The levels of the following haematological indices: WBC and Platelets were significantly lower in the test community than in the control community while haemoglobin level was significantly lower in subjects from Imiringi with reference to those from Odi. Prothrombin time was significantly increased in the test community.

The findings therefore suggest that chronic exposure to Cadmium, Chromium, Lead, Mercury and Selenium may result in oxidative stress which is an indicator for likely risk for occurrence of diseases associated with oxidative damage such as atherosclerosis, respiratory disorders, neurodegenerative diseases, such as Alzheimer's and Parkinson's disease, cancer, diabetes mellitus and inflammatory diseases among residents in Imiringi community. 


\section{International Journal of Science and Research (IJSR) \\ ISSN (Online): 2319-7064}

Index Copernicus Value (2016): 79.57 | Impact Factor (2015): 6.391

\section{Recommendations}

In view of the higher risk of exposure of people living in areas where gas flaring activities are taking place and the poor regulation of oil producing activities in these places, it is strongly recommended that all safety standards and guidelines regarding safe oil exploration and production must be adhered to strictly in order to reduce and if possible avoid contamination of the environment with pollutants. A comprehensive investigation of the heavy metal content of regularly consumed foods and water should be performed.

The fact that the oxidative stress marker (8-OHdG) correlated with some heavy metal accumulation in some locations makes a case for their use in monitoring environmental pollution.

Since oxidative stress is scientifically proven to be an important mechanism for heavy metal toxicity, then the inclusion of antioxidants in the treatment of metal induced toxicity deserves further consideration.

\section{References}

[1] Kakulu, S.E. (1985). Heavy Metals in the Niger Delta: Impact of Petroleum Industry on the Baseline Levels. Ph.D Thesis University of Ibadan.

[2] Akporhonor,E.E., Iwegbue, C.M.A., Egwaikikhide, P.A and Emua, S.A. (2007). Levels of Cadmium, Lead and Mercury in Organs of some Fish Species from Warri River, Nigeria. J. Chem. Soc. Nigeria, 32 (1) 221-226.

[3] Szymańska-Chabowska, A. , Beck, A. , Poręba, R. , Andrzejak, A. and Antonowicz-Juchniewicz, J.(2009). Evaluation of DNA Damage in People Occupationally Exposed to Arsenic and Some Heavy Metals. Polish Journal Of Environmental Studies. (18): 1131-1139.

[4] Durackova, Z. (2010). Some Current Insights into Oxidative S Stress Physiology Research. (59): 459 468.

[5] Ajugwo, A.O. (2013). Negative Effects of Gas Flaring: The Nigerian Experience. Jeph 1, 6-8.

[6] Godson, R.E., Myenpali, N.A., Sridhar, K..C., (2004). Soil Quality Mean a Chemical Fertilizer industry of Port Harcourt, Nigeria. 8:19-26.

[7] Egwurugwu J.N., Nwafor A., Nwankpa P., Olorufemi O.J., Okwara J.E. (2013). Prolonged Gas Flaring and Water Quality in Obiakpu, Egbema Imo State Nigeria, International Research Journal of Medical Science. 2(4): 1-5.

[8] www.bayelsa.gov.ng Assessed July, 2017

[9] Block, C., Freyermuth, S., Beyersmann, D. and Malviya, A.N. (1992). Role of Cadmium in Activating Nuclear Protein Kinase $\mathrm{C}$ and the Enzyme Binding to Nuclear Protein. Journal of Biology and Chemistry (28):19824-19828.

[10] Valko, M., Leibfritz, D., Moncol, J., Cronin, M.T.D., Mazur, M., Telser, J. (2007). Free Radicals and Antioxidants in Normal Physiological Functions and Human Disease. International Journal of Biochemistry and Cell Biology (23): 209-213.
[11] Saito, S., Yamauchi, H., Hasul., Y., Kurashige, J., Ochi and Yoshida, K. (2000). Quantitative Determination of 8-Hydroxydeoxyguanosine by Using ELISA, Research Communications in Molecular Pathology and Pharmacology (107): 39-44.

[12]Reitman, $S$ and Frankel, S (1957) Alanine Aminotransferase American Journal of Clinical Pathology (28): 56.

[13] Doumas, B. T., Watson, W.A, and Briggs, H.G. (1971). Albumin Clinica Chimica Acta (31): 87-96.

[14] Kochmar, J.F. and Moss, D.W. (1976). Fundamentals of Clinical Chemistry Tietz Edition. W.B. Saunders and Company, Philadelphia: 604.

[15] Jendrassik, L. and Grof, P. (1938). Bilirubin, Biochemistry (81): 297.

[16] Slot, C. (1965). Scandinevia Journal of Clinical Laboratory Investigations. (17): $381-387$.

[17] Vanencia, A., Arulselvi, S., Kanchana, R. and Ravindra, M.P. (2011). Agreement of Two Different Laboratory Methods Used to Measure Electrolytes. Journal of Laboratory Physicians (2): 104-109.

[18] Whisler, S and Dahlgren, C. (2005). Performance Evaluation of the Sysmex poch-100i Automated Haematology Analyzer Laboratory Haematology (2): 101-117.

[19] Dacie, J.V and Lewis, S.M. (1984).Practical Hematology: 120.

[20] Friberg, I. (1995). Cadmium in the Environment, CRC Press, Cleveland: $56-58$.

[21] Egwurugwu J.N., Nwafor A. , Oluronfemi O.J.. Iwuji S.C. and Alagwu E.A.(2013). Impact of Prolonged Exposure to Oil and Gas Flares on Human Renal Functions. International Research Journal of Medical Science.. 1(11): 9-16.

[22] Nwankwo C.N. and Ogagarue D.O. (2011). Effects of Gas Glaring on Surface and Ground Waters in Delta State Nigeria, Journal of Geology and Mining Research, 3(5): 131-136.

[23] Sirivarasai, J., Kaojaren, S., Wananukul, W., Srisomerang, P. (2002). Non-Occupational Determinants of Cadmium and Lead in Blood and Urine Among a General Population in Thailand. Southeast Asian Journal of Tropical Medicine and Public Health, 33(1): 180-187.

[24] Khassouani, C.E., Soulaymani, R., Mauras, Y., Allain, P. (2000). Blood Cadmium Concentration in the Population of the Rabat Area, Morocco. Clinica Chimica Acta, 302(1-2): 155-160.

[25] Bushnik, T., Haines, D., Levallois, P., Levesque, J., Van Oostdam, J., Viau, C.(2010). Lead and bisphenol A concentrations in the Canadian population. Health Reports, 21(3): 1-12.

[26] Ibeto, C.N., Okoye, C.O.B. (2010). High levels of Heavy Metalsin Blood of the Urban Population of Nigeria. Research Journal of Environmental Science (4): 371-382.

[27] Olsson, I.M., Bensryd, I., Lundh, T., Ottosson, H., Skerfving, S., Oskarsson, A.(2002). Cadmium in Blood and Urine - Impact of Sex, Age, Dietary Intake, Iron Status, and Former Smoking - Association of Renal Effects. Environmental Health Perspective, 110(12): 1185-1190. 
[28] Adedara, I.A, Ebokaiwe, A.P and Farombi, E.O (2011). Tissues Distribution of Heavy Metals and Erythrocytes Antioxidant Status in Rats Exposed to Nigerian Bonny Light Crude Oil. Toxicology and Industrial Health: 1-7.

[29] Yang, Y., Tian Y., Yan C., Jin, X., Tang, J. and Shen, X. (2008). Determinants of Urinary 8-Hydnoxyl-2Deoxyguanosine in Chinese Children with Acute Leukemia, Environmental Toxicology. (16): 128 133.

[30] Achparaki, M., Thessalonikeos, E., Tsoukali, H., Mastrogianni, O., Zaggelidou E., Chatzinikolaou, F., Vasilliades, N and Raikos, N (2012). Heavy Metals Toxicity. Aristotle University Medical Journal 39(1): $29-34$.

[31]Anslem, O.A. (2013). Negative Effects of Gas Flaring: The Nigerian Experience. Journal of Environmental Pollution and Health, (1): 6-8

\section{Author Profile}

Ifenkwe John Chimerenka is in the Chemical Pathology Department, Health Check Services, Yenagoa, Bayelsa State, Nigeria.

Nwanjo Harrison Ugochukwuis in the Department of Medical Laboratory Science, Imo State University, Owerri, Nigeria.

Nwosu Dennis Chimezie is in the Department of Medical Laboratory Science, Imo State University, Owerri, Nigeria

Ofor Igri Bassey is in the Chemical Pathology Department, Federal Medical Centre, Yenagoa, Bayelsa State, Nigeria 